\title{
Classification of Adolescent Psychiatric Patients at High Risk of Suicide Using the Personality Assessment Inventory by Machine Learning
}

\author{
Kyung-Won Kim ${ }^{1 \star}$, Jae Seok Lim ${ }^{2 \star}$, Chan-Mo Yang ${ }^{\bowtie}$, Seung-Ho Jang ${ }^{1}$, and Sang-Yeol Lee ${ }^{1}$ \\ ${ }^{1}$ Department of Psychiatry, School of Medicine, Wonkwang University, Iksan, Republic of Korea \\ ${ }^{2}$ Department of Oral and Maxillofacial Surgery, Chungbuk National University Hospital, Cheongju, Republic of Korea
}

\begin{abstract}
Objective There are growing interests on suicide risk screening in clinical settings and classifying high-risk groups of suicide with suicidal ideation is crucial for a more effective suicide preventive intervention. Previous statistical techniques were limited because they tried to predict suicide risk using a simple algorithm. Machine learning differs from the traditional statistical techniques in that it generates the most optimal algorithm from various predictors.

Methods We aim to analyze the Personality Assessment Inventory (PAI) profiles of child and adolescent patients who received outpatient psychiatric care using machine learning techniques, such as logistic regression (LR), random forest (RF), artificial neural network (ANN), support vector machine (SVM), and extreme gradient boosting (XGB), to develop and validate a classification model for individuals with high suicide risk.

Results We developed prediction models using seven relevant features calculated by Boruta algorithm and subsequently tested all models using the testing dataset. The area under the ROC curve of these models were above 0.9 and the RF model exhibited the best performance.

Conclusion Suicide must be assessed based on multiple aspects, and although Personality Assessment Inventory for Adolescent assess an array of domains, further research is needed for predicting high suicide risk groups. Psychiatry Investig 2021;18(11):1137-1143
\end{abstract}

Keywords Suicide; Child and adolescent psychiatry; Machine learning; Personality Assessment Inventory-Adolescent.

\section{INTRODUCTION}

Suicide is understood as a series of actions including suicidal ideation, suicidal planning, and suicide attempt. ${ }^{1}$ Suicidal ideation refers to immersion into thoughts about suiciderelated behaviors, ${ }^{2}$ and suicidal planning refers to specific planning to attempt suicide, which can lead to suicide attempt and ultimately death. If suicidal ideation is focused on the aspect of thinking, suicide attempt is the outward expression as a specific action. Suicide attempt includes suicide behaviors committed without the intention of suicide to achieve other

Received: June 4, 2021 Revised: September 11, 2021

Accepted: October 25, 2021

$\triangle$ Correspondence: Chan-Mo Yang, MD

Department of Psychiatry, Wonkwang University Hospital, 895 Muwang-ro, Iksan 54538, Republic of Korea

Tel: +82-63-859-1044, Fax: +82-63-857-1043, E-mail: ychanmo@wku.ac.kr

*These authors contributed equally to this work.

(a) This is an Open Access article distributed under the terms of the Creative Commons Attribution Non-Commercial License (https://creativecommons.org/licenses/by$\mathrm{nc} / 4.0$ ) which permits unrestricted non-commercial use, distribution, and reproduction in any medium, provided the original work is properly cited. goals as well as cases in which an individual attempted to kill oneself but failed.

According to OECD suicide statistics, Korea had the highest suicide rate among $35 \mathrm{OECD}$ countries in 2017 at 23.0 per 100,000 population. ${ }^{3}$ The 2019 suicide statistics published by Statistics Korea showed that suicide is the leading cause of death among individuals aged $10-39$ years, ${ }^{4}$ and suicide has continuously been the leading cause of death among the teens between the age 10-19 since 2008.

Not all adolescents with suicidal ideation actually attempt suicide, and groups of adolescents without suicidal planning may attempt to commit suicide. ${ }^{5}$ However, because suicidal ideation is highly likely to progress to suicide attempt and suicidal death, suicide attempts can be reduced by reducing suicidal ideation. ${ }^{6}$ In fact, approximately one-third of adolescents with suicidal ideation are known to make a suicide attempt, ${ }^{5}$ and preventive interventions are crucial because suicidal ideation is an important predictor of suicide attempt. ${ }^{78}$ Hence, there are growing interests on suicide risk screening in clini- 
cal settings, and classifying high-risk groups of suicide with suicidal ideation is crucial for a more effective suicide preventive intervention. ${ }^{9}$

There are several factors that influence adolescents' suicide risk, ${ }^{10}$ and specifically personality trait is one such risk factor. ${ }^{11}$ Some past studies have analyzed features of groups with a suicide risk using the Personality Assessment Inventory (PAI), ${ }^{12,13}$ but these studies were limited to adult subjects, with little relevant research on children and adolescents according to our literature review. In addition, most studies simply examined the association of suicide risk with symptoms such as depression and anxiety or identified relevant risk factors. ${ }^{14}$ Recently, some studies have applied machine learning in the prediction of suicide, ${ }^{15}$ and there was an attempt to classify or predict adolescents with a suicide risk using machine learning. ${ }^{15-17}$ However, none of the studies attempted to predict adolescents with high suicide risk using machine learning in consideration of their personality traits. Thus, this study aims to classify groups at risk of suicide by including personality traits using the PAI.

Previous statistical techniques were limited because they tried to predict suicide risk, a complex problem, using a simple algorithm..$^{18}$ Machine learning differs from the traditional statistical techniques in that it generates the most optimal algorithm from various predictors, based on which it has been proposed to be useful in the prediction and classification of suicide risk. ${ }^{19}$ In the present study, we aim to analyze the PAI profiles of child and adolescent patients who received outpatient psychiatric care using machine learning techniques, such as logistic regression (LR), random forest (RF), artificial neural network (ANN), support vector machine (SVM), and extreme gradient boosting (XGB), to develop and validate a classification model for individuals with high suicide risk.

\section{METHODS}

\section{Participants}

Data were obtained from retrospective chart review of psychometric assessment, which was performed on 158 patients aged 12-17 years who have visited the outpatient psychiatric clinic at Wonkwang University hospital between January 2011 and December 2019. Overall psychological evaluation including PAI-A and intelligence test were conducted as initial assessments if it has not been evaluated before. Because the reliability of self-reported data from individuals with an overall intelligence and language comprehension score of below 70 is low, 34 were excluded, and a total of 124 patients were enrolled. The psychometric assessment results were interpreted by a clinical psychologist. The diagnosis of all patients was grouped according to DSM-IV-TR and DSM-5. This study was approved by the Institutional Review Board of Wonk- wang University Hospital (WKUH 2021-02-013-004).

\section{Instruments}

Personality Assessment Inventory for Adolescent (PAI-A)

This test is an objective test for assessing personality and psychopathology developed by Morey ${ }^{20}$ and standardized for use in Korea by Kim et al. ${ }^{21}$ The test was developed to provide important information about the patient or client in clinical settings, and according to the standardization study, it is a useful multiscale inventory personality test that enables differential diagnosis and identifies specific areas of discomfort among individuals. The PAI-A retains the same scale structure in the PAI, and it was developed for use on middle and high school students by modifying items that were deemed to be inappropriate for adolescents. It comprises 264 items, with 22 scales, including four validity scales, 11 clinical scales, five treatment consideration scales, and two interpersonal scales. Ten of these scales include subscales designed to facilitate a comprehensive and in-depth assessment of complex clinical constructs.

Lim et al. ${ }^{22}$ conducted a restandardization study of Korean PAI-A in 2018, and the internal consistency of each scales were identified as NIM (Cronbach's $\alpha=0.76)$, PIM $(\alpha=0.75)$, $\operatorname{SOM}(\alpha=0.80), \operatorname{ANX}(\alpha=0.84), \operatorname{ARD}(\alpha=0.70), \operatorname{DEP}(\alpha=0.87)$, $\operatorname{MAN}(\alpha=0.74), \operatorname{PAR}(\alpha=0.75), \operatorname{SCZ}(\alpha=0.80), \operatorname{BOR}(\alpha=0.88)$, ANT ( $\alpha=0.77)$, ALC ( $\alpha=0.51), \operatorname{DRG}(\alpha=0.64), \operatorname{AGG}(\alpha=0.80)$, SUI $(\alpha=0.82)$, STR $(\alpha=0.79)$, NON $(\alpha=0.66)$, RXR $(\alpha=0.73)$, $\operatorname{DOM}(\alpha=0.67)$ and WRM $(\alpha=0.80)$.

SUI scale is related to evaluation of idea of death and suicide, with little thought of death or suicide if it is below 60 points. And with 60-69 points, there are temporary and periodic suicidal ideation and tend to be pessimistic about the future of one self, and a significant sense of suicide is reported if the score is 70 or higher. In this study, subjects with a SUI scale of 60 or higher were defined as suicide high risk group.

\section{Machine learning}

The prediction pipeline was developed as shown in Figure 1. The pipeline was generated from five machine learning methods, namely LR, RF, ANN, SVM, and XGB using the caret package provided in the $\mathrm{R}$ statistical software version 3.6.3 ( $\mathrm{R}$ Studio, Inc., Boston, MA, USA). The developed pipeline consisted of random splitting of the input dataset into training ( $\mathrm{n}=87 ; 70 \%$ of 124 samples) and testing $(\mathrm{n}=37,30 \%$ of 124 samples) datasets, while maintaining equal proportions of the class ratios in each split. We developed five final machine learning models to predict high suicide risk group in the training dataset, by tuning the hyper-parameters using the caret package provided with the $\mathrm{R}$ statistical software. We used ten-fold 


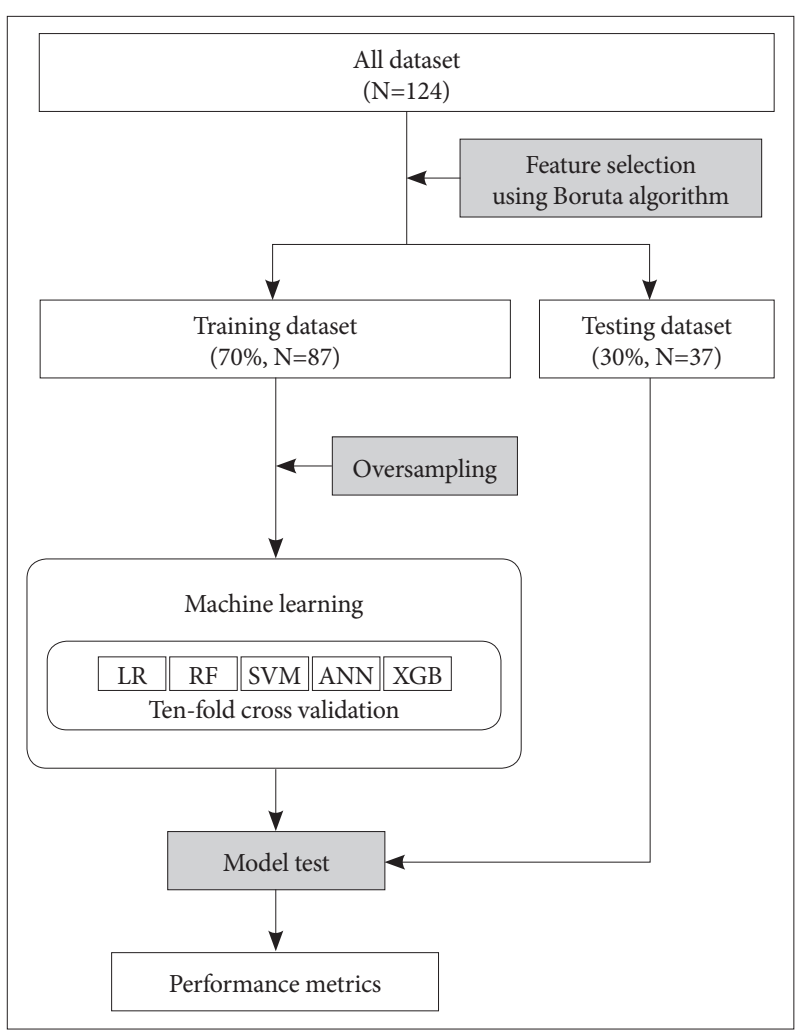

Figure 1. Schematic of prediction model development. LR, logistic regression; RF, random forest; ANN, artificial neural network; SVM, support vector machine; XGB, extreme gradient boosting.

cross-validation to prevent overfitting. The relative importance of feature, provided in arbitrary units, was calculated using the Boruta algorithm, which is a variable selection method built around the random forest. ${ }^{23}$ The receiver operating characteristic (ROC) curves were plotted, and the area under the ROC curves (AUROC) was obtained to assess the model's performance. The AUROCs were compared using the Delong test.

\section{Statistical analysis}

Statistical analysis was conducted using the R software ( $R$ Studio). The demographic data were analyzed using student's t-test and $\chi^{2}$ test, as appropriate. $\mathrm{p}$-values $<0.05$ (two-sided) were considered statistically significant.

\section{RESULTS}

\section{Differences of demographic characteristics and intelligence scores between high-risk and low-risk groups}

A total of 124 participants were divided into the high suicide risk group (suicide ideation, SUI $\geq 60$ ) or low suicide risk group (SUI <60) based on their SUI score. There were no significant differences in the demographic factors and intelligence scores between the two groups (Table 1).
Table 1. Demographic characteristics and intelligence scores

\begin{tabular}{|c|c|c|c|}
\hline Dependent: suicide risk & $\begin{array}{c}\text { High-risk } \\
(\mathrm{N}=44)\end{array}$ & $\begin{array}{c}\text { Low-risk } \\
(\mathrm{N}=80)\end{array}$ & $\mathrm{p}$ \\
\hline Sex & & & $>0.999$ \\
\hline Female & $19(43.2)$ & $34(42.5)$ & \\
\hline Male & $25(56.8)$ & $46(57.5)$ & \\
\hline Age (years) & $15.1 \pm 1.9$ & $14.9 \pm 1.5$ & 0.528 \\
\hline Living with both parents & & & 0.122 \\
\hline Yes & $30(68.1)$ & $65(81.2)$ & \\
\hline No & $14(31.9)$ & $15(18.8)$ & \\
\hline Education statement & & & 0.867 \\
\hline Middle school & $21(47.8)$ & $42(52.5)$ & \\
\hline High school & $22(50.0)$ & $36(45.0)$ & \\
\hline Dropping out & $1(2.2)$ & $2(2.5)$ & \\
\hline Verbal comprehensive index & $90.1 \pm 14.7$ & $88.2 \pm 16.3$ & 0.527 \\
\hline Working memory index & $95.0 \pm 19.5$ & $94.2 \pm 17.7$ & 0.816 \\
\hline Perceptual reasoning index & $89.4 \pm 15.8$ & $94.1 \pm 16.9$ & 0.138 \\
\hline Processing speed index & $86.0 \pm 19.3$ & $88.2 \pm 15.7$ & 0.513 \\
\hline Full-Scale IQ & $86.9 \pm 18.0$ & $87.5 \pm 16.3$ & 0.862 \\
\hline \multicolumn{4}{|l|}{ Diagnosis } \\
\hline Psychotic disorder & $1(2.3)$ & $4(5.0)$ & 0.460 \\
\hline Mood disorder & $14(31.8)$ & $26(32.5)$ & 0.938 \\
\hline Anxiety disorder & $17(38.6)$ & $25(31.3)$ & 0.406 \\
\hline $\mathrm{ADHD}$ & $6(13.6)$ & $10(12.5)$ & 0.857 \\
\hline Tic disorder & $2(4.5)$ & $6(7.5)$ & 0.522 \\
\hline Conduct/ODD & $6(13.6)$ & $6(7.5)$ & 0.269 \\
\hline Others & $4(9.1)$ & $11(13.8)$ & 0.447 \\
\hline
\end{tabular}

Data are presented as mean \pm standard deviation or $\mathrm{N}(\%)$. ADHD, attention deficit hyperactivity disorder; ODD, oppositional defiant disorder

\section{Comparison of PAI-A scale scores according to suicide risk}

There were statistically significant differences in the inconsistency (ICN) scale, negative impression (NIM) scale, positive impression (PIM) scale, somatic complaints (SOM) scale, anxiety (ANX) scale, anxiety-related disorder (ARD) scale, depression (DEP) scale, mania (MAN) scale, paranoia (PAR) scale, schizophrenia (SCZ) scale, borderline features (BOR) scale, drug problems (DRG) scale, aggression (AGG) scale, stress (STR) scale, nonsupport (NON) scale, and treatment rejection (RXR) scale of PAI-A between the two groups. There were no statistically significant differences in the infrequency (INF) scale, antisocial features (ANT) scale, alcohol problems (ALC) scale, dominance (DOM) scale, and warmth (WRM) scale between the two groups (Table 2). 
Table 2. Comparison of PAI-A scale by suicide risk

\begin{tabular}{|c|c|c|c|}
\hline Dependent: suicide risk & High-risk & Low-risk & $\mathrm{p}$ \\
\hline $\mathrm{ICN}$ & $51.0 \pm 9.6$ & $47.5 \pm 9.1$ & 0.043 \\
\hline INF & $52.3 \pm 10.9$ & $55.1 \pm 8.3$ & 0.112 \\
\hline NIM & $64.5 \pm 13.7$ & $53.6 \pm 12.8$ & $<0.001$ \\
\hline PIM & $41.7 \pm 10.7$ & $48.2 \pm 11.7$ & 0.003 \\
\hline SOM & $62.3 \pm 18.0$ & $53.9 \pm 13.5$ & 0.004 \\
\hline ANX & $59.7 \pm 12.2$ & $47.6 \pm 10.4$ & $<0.001$ \\
\hline ARD & $61.7 \pm 11.2$ & $46.5 \pm 9.8$ & $<0.001$ \\
\hline DEP & $69.8 \pm 13.5$ & $57.5 \pm 14.9$ & $<0.001$ \\
\hline MAN & $48.8 \pm 14.3$ & $42.8 \pm 11.9$ & 0.014 \\
\hline PAR & $61.9 \pm 15.0$ & $55.7 \pm 13.7$ & 0.021 \\
\hline SCZ & $59.9 \pm 12.2$ & $59.9 \pm 12.2$ & 0.001 \\
\hline BOR & $62.8 \pm 12.6$ & $53.4 \pm 14.2$ & $<0.001$ \\
\hline ANT & $58.6 \pm 15.5$ & $54.8 \pm 13.4$ & 0.158 \\
\hline ALC & $54.5 \pm 14.6$ & $53.2 \pm 11.9$ & 0.591 \\
\hline DRG & $55.2 \pm 12.2$ & $50.9 \pm 9.8$ & 0.032 \\
\hline AGG & $58.5 \pm 13.6$ & $51.9 \pm 11.9$ & 0.005 \\
\hline STR & $60.5 \pm 10.9$ & $49.1 \pm 10.7$ & $<0.001$ \\
\hline $\mathrm{NON}$ & $61.6 \pm 11.9$ & $1.4 \pm 11.7$ & $<0.001$ \\
\hline RXR & $43.4 \pm 14.8$ & $51.2 \pm 11.6$ & 0.002 \\
\hline DOM & $44.7 \pm 9.9$ & $43.9 \pm 12.2$ & 0.724 \\
\hline WRM & $48.0 \pm 13.3$ & $45.2 \pm 11.1$ & 0.208 \\
\hline
\end{tabular}

Data are presented as mean \pm standard deviation. PAI-A, Personality Assessment Inventory for Adolescent; ICN, inconsistency; INF, infrequency; NIM, negative impression; PIM, positive impression; SOM, somatic complaints; ANX, anxiety; ARD, anxiety-related disorders; DEP, depression; MAN, mania; PAR, paranoia; SCZ, schizophrenia; BOR, borderline features; ANT, antisocial features; ALC, alcohol problems; DRG, drug problems; AGG, aggression; STR, stress; NON, nonsupport; RXR, treatment rejection; DOM, dominance; WRM, warmth

\section{Develop a prediction model using machine learning techniques}

The observed high-risk ratio was $35.5 \%$ (44/124), which was consistent with the imbalanced data (Table 1). Therefore, we applied the oversampling method to balance the training dataset (Figure 1). First, we developed the prediction models with sex, age and all PAI-A scales and subsequently tested all models using the testing dataset. The AUROCs of RF, SVM and XGB were $>0.8$, indicating that these models performed effectively in the testing dataset (Figure 2). Then, the relative importance of all features was calculated using the Boruta algorithm. Seven features including ARD, NON, DEP, RXR, STR, ANX, and AGG scales were determined as relevant for predicting high suicide risk group and the ARD showed the highest relative importance (Figure 3). Finally, we developed prediction models using seven relevant features calculated by Boruta algorithm and subsequently tested all models using

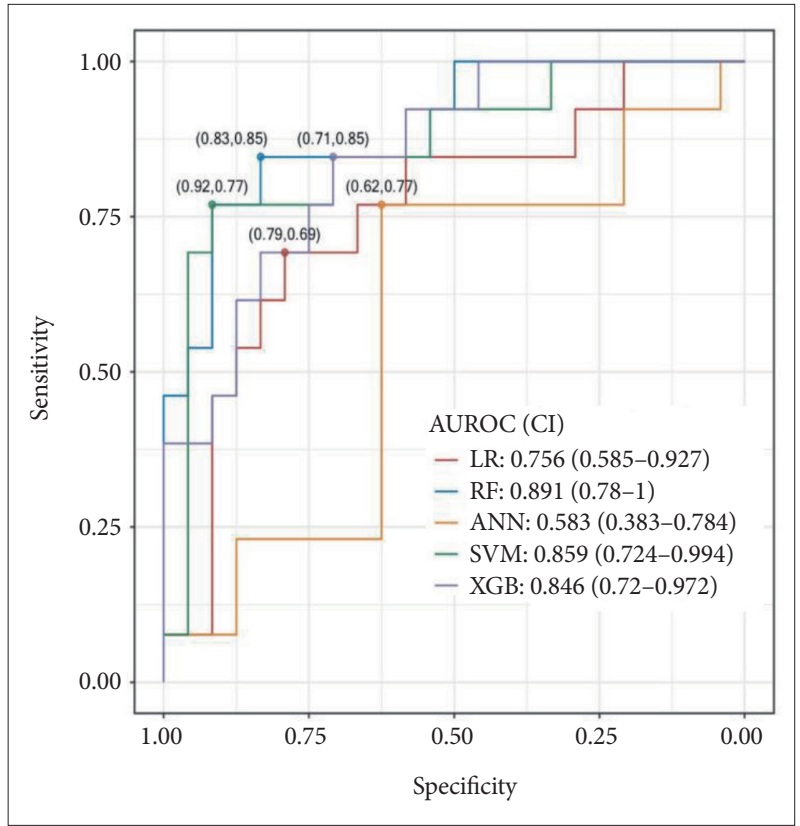

Figure 2. Receiver operating characteristic curves plotted from machine learning models developed with sex, age, and Personality Assessment Inventory for Adolescent scales. AUROC, area under the ROC curve; $\mathrm{Cl}$, confidence interval; LR, logistic regression; RF, random forest; ANN, artificial neural network; SVM, support vector machine; XGB, extreme gradient boosting.

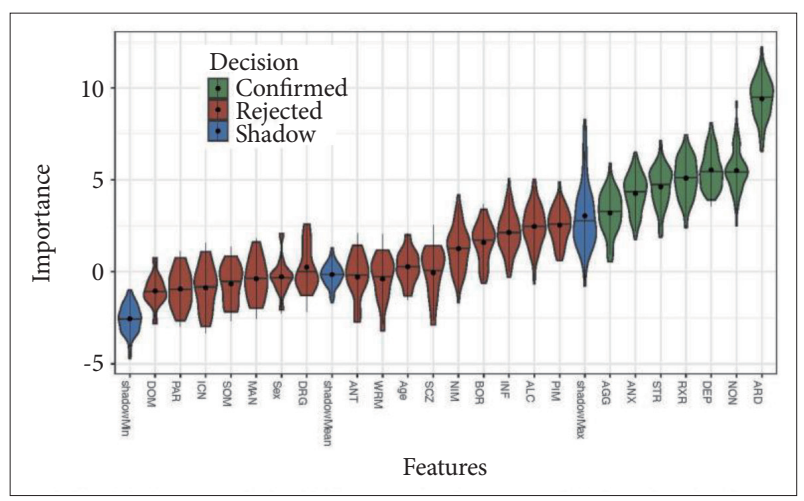

Figure 3. Relative feature importance computed using the Boruta algorithm. The blue violin plots correspond to the minimal, average, and maximum $Z$ scores of a shadow attribute. The red and green violin plots represent the $Z$ scores of the rejected and confirmed attributes, respectively. The black dots and horizontal lines inside each violin plot represent the mean and median values, respectively. All features that received a lower relative feature importance than that of the shadow feature were defined as irrelevant for prediction. Laterality was considered an irrelevant feature (marked in red). ICN, inconsistency; INF, infrequency; NIM, negative impression; PIM, positive impression; SOM, somatic complaints; ANX, anxiety; ARD, anxiety-related disorders; DEP, depression; MAN, mania; PAR, paranoia; SCZ, schizophrenia; BOR, borderline features; ANT, antisocial fea-tures; ALC, alcohol problems; DRG, drug problems; AGG, aggression; STR, stress; NON, nonsupport; RXR, treatment rejection; DOM, dominance; WRM, warmth.

the testing dataset. The AUROCs of these models were above 0.9 and the RF model exhibited the best performance (Figure 4 and Table 3). The performance of RF model was significant- 


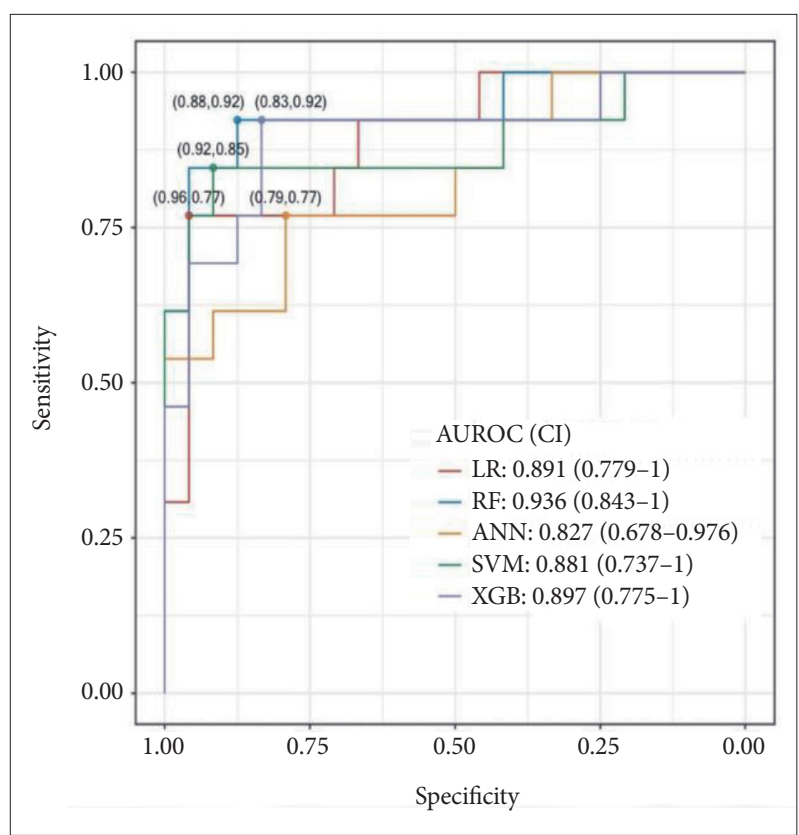

Figure 4. Receiver operating characteristic curves plotted from machine learning models developed with selected features by Boruta algorithm. AUROC, area under the ROC curve; $\mathrm{Cl}$, confidence interval; LR, logistic regression; RF, random forest; ANN, artificial neural network; SVM, support vector machine; XGB, extreme gradient boosting.

Table 3. Performance metrics of the prediction models

\begin{tabular}{lcccccc}
\hline Model & Accuracy & Sensitivity & Specificity & PPV & NPV & F1 \\
\hline LR & 0.89 & 0.77 & 0.96 & 0.91 & 0.88 & 0.83 \\
RF & 0.89 & 0.92 & 0.88 & 0.80 & 0.95 & 0.86 \\
ANN & 0.78 & 0.77 & 0.79 & 0.67 & 0.86 & 0.71 \\
SVM & 0.89 & 0.85 & 0.92 & 0.85 & 0.92 & 0.85 \\
XGB & 0.86 & 0.92 & 0.83 & 0.75 & 0.95 & 0.83 \\
\hline
\end{tabular}

LR, logistic regression; RF, random forest; $A N N$, artificial neural network; SVM, support vector machine; XGB, extreme gradient boosting; PPV, positive predictive value; NPV, negative predictive value; F1, F1-score

ly superior to that of ANN (Table 4).

\section{DISCUSSION}

This study attempted to develop and validate a model for predicting high suicide risk in child and adolescent psychiatric patients using the scales of PAI-A and applying machine learning techniques.

In this study, approximately $35.5 \%(n=44)$ of the participants were classified as the high suicide risk group, as defined by suicide ideation scale score of 60 or higher. Jeon et al. ${ }^{24}$ reported the lifetime prevalence of suicidal ideation to be $18.9 \%$ among adolescents aged $12-18$ years, and the higher prevalence in our study may be attributable to the fact that the study
Table 4. Calculated probability of the difference between the area under the receiver operating characteristic curve

\begin{tabular}{lllll}
\hline \multicolumn{1}{c}{ Model } & RF & ANN & SVM & XGB \\
\hline LR & 0.872 & 0.057 & 0.417 & 0.551 \\
RF & & $0.034^{*}$ & 0.083 & 0.068 \\
ANN & & & 0.836 & 0.858 \\
SVM & & & & 0.633 \\
\hline
\end{tabular}

${ }^{*} \mathrm{p}<0.05$. LR, logistic regression; RF, random forest; ANN, artificial neural network; SVM, support vector machine; XGB, extreme gradient boosting; DeLong's test was used

population consisted of patients who sought psychiatric care, who have various risk factors and psychopathologies, as opposed to the general population.

According to a study comparing the psychometric variables of high and low suicide risk groups by Heo et al., ${ }^{25}$ the high suicidal ideation group had significantly higher scores on the clinical scales of PAI, namely SOM, ANX, ARD, DEP, MAN, PAR, BOR, ANT, and DRG scales, compared to the low suicidal ideation group. In the study of suicide attempt by Sinclair et al., ${ }^{12}$ total nine indicators (ICN, NIM, STR, MAN-G, SCZ-T, BOR-N, BOR-S, ANT-A, and ANT-E) among 42 PAI indicators and specifically six indicators (NIM, STR, MANG, BOR-N, BOR-S, ANT-A) when considering several factors such as age, sex, education, and race were found to differ between multiple suicide attempt and single/no suicide attempt. The scales found to be significantly associated with suicide risk classification in our study, namely ARD, NON, DEP, ANX, RXR, AGG, and STR scales, differed from those reported by previous studies, and this is presumed to be due to the adolescent study population in our study and application of machine learning methods.

AGG was found to be a significant factor in classifying high suicide risk group. It has been suggested as a developmental factor of suicide in a previous study, ${ }^{26}$ and it has also been reported to be associated with ADHD, ODD, and conduct disorders among children and adolescents, with adolescents with a suicide attempt history showed higher impulsivity than those without a suicide attempt history. ${ }^{27}$ In the developmental aspect of neuromaturation, adolescent's aggression can be interpreted in the context that structural and functional maturation of the prefrontal cortex is in progress, and it is still difficult to demonstrate consistent impulse control. ${ }^{28,29}$ Adolescents with conduct disorder showed a high suicide risk, and some studies have linked this to severe emotional dysregulation and higher prevalence of mood disorder. ${ }^{30}$ Substance abuse is also a risk factor of suicide attempt in adolescents, and one study reported that heavy drinking increases psychological distress and aggressiveness and hinders adaptive coping strategy, thereby increasing suicide risk. ${ }^{31}$ 
NON scale indicates a lack of social support, and this scale can be used to examine the degree of support provided by family members and friends. A previous study reported that female adolescents who are socially severed from their friends engage in more suicidal ideation, while male adolescents who belong in a tightly networked school community exhibit a protective effect against suicide attempt. ${ }^{32}$ Furthermore, this is in line with the findings that family stress or familial conflict directly and indirectly influence adolescents' suicide risk behaviors. ${ }^{33-35}$

Anxiety disorder and PTSD are known to increase suicide risk. ${ }^{26}$ In addition, patients with depression are 13-26 times higher risk of suicide compared to the general population, ${ }^{36}$ and a psychological autopsy study on 229 suicidal deaths found that $59 \%$ of these people had major depressive disorder. ${ }^{37}$ In our study, anxiety as well as depression were identified as risk factors for suicide, in this regard previous study ${ }^{38}$ shown that the coexistence of depressive and anxiety symptoms was associated with an increased risk of suicide compared to having either one.

RXR evaluate the interest in psychological and emotional change, indicating that individual with high scores lack therapeutic motivation. According to a study of untreated adolescent depression, ${ }^{39}$ those who were not treated were found to have 4.19 times higher risk of suicide than those who were treated or who were the first depressive episode.

This study has several strengths. First, the study population comprised child and adolescent psychiatric patients. Second, data were analyzed using machine learning techniques. Suicide is a complex psychological phenomenon that occurs as a result of numerous factors. A past study confirmed the importance of machine learning techniques compared to other existing methodologies that utilize data in testing a priori hypothesis when testing the interactions among various complex factors, ${ }^{19}$ and the predictive accuracy was higher with machine learning techniques. In this study, we developed and validated a classification model for high suicide risk group using various psychopathologies, including personality traits with a standardized instrument. There were no studies that analyzed the risk factors of suicide in adolescents by both assessing them under a single standardized tool that can evaluate comorbid psychopathologies and applying machine learning techniques. The Boruta algorithm has an advantage of eliminating researcher's intention in feature selection, and we were able to confirm that the predictive model has better performance in such case. Third, in light of past studies on the association between suicide risk and intelligence, we set an intelligence score of 70 or higher as an inclusion criterion and included intelligence in the high suicide risk classification model for analysis. A previous study reported an inverse as- sociation between IQ and suicide risk in children and adolescents, and it has been found that those with a low IQ who have no appropriate coping and cognitive skills are vulnerable to in suicidal ideation upon a stressful life event, as a result suicidal ideation and planning ultimately leads to a suicide attempt. ${ }^{40,41}$

There are some limitations to this study. The first one is limited generalizability since subjects in this study has been selected among patient group who have visited child and adolescent psychiatric clinic in university hospital. Second, we used a self-report test. A retrospective self-report test is vulnerable to recall bias or underreporting. Subsequent studies should supplement the self-reported data with structured interviews. Third, causation cannot be drawn due to the crosssectional design. However, this study has clinical significance in that various facets of personality and psychopathology related to suicide risk were included in the analysis.

In conclusion, this study developed a model to predict high suicide risk groups by applying machine learning techniques to PAI-A data. The model had an AUC of 0.936, confirming its potential for classifying and predicting high suicide risk groups with a "excellent" diagnostic accuracy. ${ }^{42}$ In comparison to previous study findings using PAI, AGG, NON, and RXR were added as an important consideration in this study, and it may be helpful to detect suicides risk if these scales are actually elevated in clinical settings. However, suicide must be assessed based on multiple aspects, and although PAI-A assess an array of domains, further research is needed for predicting high suicide risk groups. In addition, large-scale data obtained from multiple institutions and further research will be needed to enhance the performance of the developed model and improves its applicability in clinical settings. Such model would be used for assessing and screening patients with high suicide risk in clinical practice.

\section{Availability of Data and Material}

The datasets generated or analyzed during the study are available from the corresponding author on reasonable request.

\section{Conflicts of Interest}

The authors have no potential conflicts of interest to disclose.

\section{Author Contributions}

Conceptualization: Chan-Mo Yang. Data curation: Kyung-Won Kim, Chan-Mo Yang. Formal analysis: Kyung-Won Kim, Jae Seok Lim. Investigation: Kyung-Won Kim, Chan-Mo Yang. Methodology: Kyung-Won Kim, Jae Seok Lim, Chan-Mo Yang. Project administration: Chan-Mo Yang. Resources: Chan-Mo Yang. Supervision: Sang-Yeol Lee. Validation: Chan-Mo Yang, Seung-Ho Jang. Visualization: Jae Seok Lim. Writing_original draft: Kyung-Won Kim, Jae Seok Lim. Writing-review \& editing: Kyung-Won Kim, Chan-Mo Yang.

\section{ORCID iDs}

Kyung-Won Kim https://orcid.org/0000-0002-7339-478X Jae Seok Lim https://orcid.org/0000-0001-5755-7529 
Chan-Mo Yang

Seung-Ho Jang

Sang-Yeol Lee

https://orcid.org/0000-0002-4959-7595

https://orcid.org/0000-0002-3479-0552

https://orcid.org/0000-0003-1828-9992

\section{Funding Statement}

This paper was supported by Wonkwang University in 2021.

\section{REFERENCES}

1. Ladame F, Jeanneret O. Suicide in adolescence: some comments on epidemiology and prevention. J Adolesc 1982;5:355-366.

2. O'Carroll PW, Berman AL, Maris RW, Moscicki EK, Tanney BL, Silverman MM. Beyond the Tower of Babel: a nomenclature for suicidology. Suicide Life Threat Behav 1996;26:237-252.

3. OECD. Suicide rate. 2017. Available at: https://data.oecd.org/healthstat/suicide-rates.htm. Accessed May 27, 2021.

4. Statistics Korea. Causes of death statistics in 2019. Available at: http:// kostat.go.kr/assist/synap/preview/skin/doc. html?fn=synapview385219_1\&rs=/assist/synap/preview. Accessed May 27, 2021.

5. Nock MK, Green JG, Hwang I, McLaughlin KA, Sampson NA, Zaslavsky $\mathrm{AM}$, et al. Prevalence, correlates, and treatment of lifetime suicidal behavior among adolescents: results from the National Comorbidity Survey Replication Adolescent Supplement. JAMA Psychiatry 2013;70: 300-310.

6. Beck AT, Kovacs M, Weissman A. Assessment of suicidal intention: the Scale for Suicide Ideation. J Consult Clin Psychol 1979;47:343-352.

7. Kumar G, Steer RA. Psychosocial correlates of suicidal ideation in adolescent psychiatric inpatients. Suicide Life Threat Behav 1995;25:339346.

8. Lim JS, Yang CM, Baek JW, Lee SY, Kim BN. Prediction models for suicide attempts among adolescents using machine learning techniques. Clin Psychopharmacol Neurosci 2021. Available at: https://www.cpn. or.kr/journal/view.html?uid=1263\&vmd=Full. Accessed May 31, 2021.

9. Horowitz LM, Bridge JA, Pao M, Boudreaux ED. Screening youth for suicide risk in medical settings: time to ask questions. Am J Prev Med 2014;47:S170-S175.

10. Bilsen J. Suicide and youth: risk factors. Front Psychiatry 2018;9:540.

11. Brezo J, Paris J, Turecki G. Personality traits as correlates of suicidal ideation, suicide attempts, and suicide completions: a systematic review. Acta Psychiatr Scand 2006;113:180-206.

12. Sinclair SJ, Roche MJ, Temes C, Massey C, Chung WJ, Stein M, et al. Evaluating chronic suicide risk with the Personality Assessment Inventory: Development and initial validation of the Chronic Suicide Risk Index (S_Chron). Psychiatry Res 2016;245:443-450.

13. Patry MW, Magaletta PR. Measuring suicidality using the Personality Assessment Inventory: a convergent validity study with federal inmates. Assessment 2015;22:36-45.

14. Nam JS, Kim DH, Kim EK. Psychological characteristics of psychiatric outpatients with high suicide risk: using MMPI-2-RF. Korean J Psychosom Med 2020;28:8-19.

15. Burke TA, Ammerman BA, Jacobucci R. The use of machine learning in the study of suicidal and non-suicidal self-injurious thoughts and behaviors: A systematic review. J Affect Disord 2019;245:869-884.

16. Jung JS, Park SJ, Kim EY, Na KS, Kim YJ, Kim KG. Prediction models for high risk of suicide in Korean adolescents using machine learning techniques. PLoS One 2019;14:e0217639.

17. Su C, Aseltine R, Doshi R, Chen K, Rogers SC, Wang F. Machine learning for suicide risk prediction in children and adolescents with electronic health records. Transl Psychiatry 2020;10:413.

18. Rozek DC, Andres WC, Smith NB, Leifker FR, Arne K, Jennings G, et al. Using machine learning to predict suicide attempts in military personnel. Psychiatry Res 2020;294:113515.

19. Walsh CG, Ribeiro JD, Franklin JC. Predicting risk of suicide attempts over time through machine learning. Clin Psychol Sci 2017;5:457-469.

20. Morey LC. The Personality Asssessment Inventory Manaual. Odessa, FL: Psychological Assessent Resources; 1991.

21. Kim YH, Kim JH, Oh SW, Lim YR, Hong SH. Standardization study of personality assessment inventory (PAI): reliability and validity. Kor J Clin Psychol 2001;20:311-329.

22. Lim SH, Hwang ST, Kweon HS, Kim JH, Park EY, Park JK, et al. Restandardization Study of the Korean Personality Assessment Inventory for Adolescent (PAI-A): Reliability and validity. Clin Psychol Kor Res Pract 2018;4:435-454.

23. Kursa MB, Rudnicki WR. Feature selection with the Boruta package. J Stat Softw 2010;36:1-13.

24. Jeon HJ, Bae JM, Woo JM. Recent statistics and risk factors of suicide in children and adolescents. J Korean Med Assoc 2013;56:93-99.

25. Heo EH, Jeong SH, Kang HY. Comparative study on Personality Assessment Inventory and MMPI-2 profiles of groups with high and low depression and suicide ideation in psychiatry patients and discriminant variables of depression and suicide ideation. J Korean Neuropsychiatr Assoc 2018;57:86-95.

26. Turecki G, Brent DA, Gunnell D, O'Connor RC, Oquendo MA, Pirkis J, et al. Suicide and suicide risk. Nat Rev Dis Primers 2019;5:1-22.

27. Charles NE, Bullerjahn MR, Barry CT. Understanding at-risk youths: average PAI-A scores and their associations with impulsivity-related constructs. J Pers Assess 2021;103:33-47.

28. Johnson SB, Blum RW, Giedd JN. Adolescent maturity and the brain: the promise and pitfalls of neuroscience research in adolescent health policy. J Adolesc Health 2009;45:216-221.

29. Blakemore SJ, Choudhury S. Development of the adolescent brain: implications for executive function and social cognition. J Child Psychol Psychiatry 2006;47:296-312.

30. Wei HT, Lan WH, Hsu JW, Bai YM, Huang KL, Su TP, et al. Risk of suicide attempt among adolescents with conduct disorder: a longitudinal follow-up study. J Pediatr 2016;177:292-296.

31. Pompili M, Serafini G, Innamorati M, Biondi M, Siracusano A, Di Giannantonio $\mathrm{M}$, et al. Substance abuse and suicide risk among adolescents. Eur Arch Psychiatry Clin Neurosci 2012;262:469-485.

32. Bearman PS, Moody J. Suicide and friendships among American adolescents. Am J Public Health 2004;94:89-95.

33. Randell BP, Wang WL, Herting JR, Eggert LL. Family factors predicting categories of suicide risk. J Child Fam Stud 2006;15:247-262.

34. Janiri D, Doucet GE, Pompili M, Sani G, Luna B, Brent DA, et al. Risk and protective factors for childhood suicidality: a US population-based study. Lancet Psychiatry 2020;7:317-326.

35. Brent DA, Maalouf FT. Pediatric depression: is there evidence to improve evidence-based treatments? J Child Psychol Psychiatry 2009;50: 143-152.

36. Angst F, Stassen H, Clayton PJ, Angst J. Mortality of patients with mood disorders: follow-up over 34-38 years. J Affect Disord 2002;68:167-181.

37. Henriksson MM, Aro HM, Marttunen MJ, Heikkinen ME, Isometsa ET, Kuoppasalmi KI, et al. Mental disorders and comorbidity in suicide. Am J Psychiatry 1993;150:935-940.

38. Melton TH, Croarkin PE, Strawn JR, McClintock SM. Comorbid anxiety and depressive symptoms in children and adolescent: a systematic review and analysis. J Psychiatr Pract 2016;22:84-98.

39. Yang CM, Lee SY. Effect of untreated depression in adolesence on the suicide risk and attempt in male young adults. Korean J Psychosom Med 2020;28:29-35.

40. Gunnell D, Harbord R, Singleton N, Jenkins R, Lewis G. Is low IQ associated with an increased risk of developing suicidal thoughts? Soc Psychiatry Psychiatr Epidemiol 2009;44:34-38.

41. Gunnell D, Magnusson PKE, Rasmussen F. Low intelligence test scores in 18 year old men and risk of suicide: cohort study. BMJ 2005;330:167.

42. Šimundić AM. Measures of diagnostic accuracy: basic definitions. EJIFCC 2009;19:203-211. 